\title{
Matrine suppresses cardiac fibrosis by inhibiting the TGF- $\beta$ /Smad pathway in experimental diabetic cardiomyopathy
}

\author{
YONG ZHANG $^{1}$, LEI CUI ${ }^{2}$, GONGCHANG GUAN ${ }^{1}$, JUNKUI WANG ${ }^{1}$, \\ CHUAN QIU ${ }^{3}$, TIELIN YANG ${ }^{4}$, YAN GUO ${ }^{4}$ and ZHONGWEI LIU ${ }^{1,4}$ \\ ${ }^{1}$ Department of Cardiology, Shaanxi Provincial People's Hospital, Xi'an, Shaanxi 710068; ${ }^{2}$ Department of \\ Ultrasonography, Xianyang Central Hospital, Xianyang, Shaanxi 712000, P.R. China; ${ }^{3}$ Department of \\ Biostatistics and Bioinformatics, School of Public Health and Tropical Medicine, Tulane University, \\ New Orleans, LA 70112, USA; ${ }^{4}$ Institute of Molecular Genetics, School of Life Science and \\ Technology, Xi'an Jiaotong University, Xi'an, Shaanxi 710000, P.R. China
}

Received March 11, 2017; Accepted October 19, 2017

DOI: $10.3892 / \mathrm{mmr} .2017 .8054$

\begin{abstract}
Cardiac fibrosis is one of the pathological characteristics of diabetic cardiomyopathy ( $\mathrm{DbCM})$. Matrine treatment has proven to be effective in cases of organ fibrosis and cardiovascular diseases. In the present study, the anti-fibrosis-associated cardioprotective effects of matrine on $\mathrm{DbCM}$ were investigated. Rats with experimental $\mathrm{DbCM}$ were administered matrine orally. Cardiac functions were evaluated using invasive hemodynamic examinations. Cardiac compliance was assessed in isolated hearts. Using Sirius Red and fluorescence staining, the collagen in diabetic hearts was visualized. MTT assay was used to select non-cytotoxic concentrations of matrine, which were subsequently used to treat isolated cardiac fibroblasts incubated under various conditions. Western blotting was performed to assess activation of the transforming growth factor- $\beta 1$ (TGF- $\beta 1$ )/Smad signaling pathway. Rats with DbCM exhibited impaired heart compliance and left ventricular (LV) functions. Excessive collagen deposition in cardiac tissue was also observed. Furthermore, TGF- $\beta 1 /$ R-Smad (Smad2/3) signaling was
\end{abstract}

Correspondence to: Dr Zhongwei Liu, Department of Cardiology, Shaanxi Provincial People's Hospital, 257 Youyi Western Road, Xi'an, Shanxi 710068, P.R. China

E-mail: liuzhongwei@xjtu.edu.cn

Abbreviations: DbCM, diabetic cardiomyopathy; ECM, extracellular matrix; CFs, cardiac fibroblasts; TGF- $\beta 1$, transforming growth factor- $\beta 1$; STZ, streptozotocin; LV, left ventricular; LVSP, left ventricular systolic pressure; LVEDP, left ventricular end-diastolic pressure; $+\mathrm{LVdP} / \mathrm{dt}_{\max }$, positive maximal rate of left ventricular increased pressure; $-\mathrm{LVdP} / \mathrm{dt}_{\max }$, negative maximal rate of left ventricular increased pressure; IGF, insulin-like growth factor; FBS, fetal bovine serum; BCA, bicinchoninic acid; PVDF, polyvinylidene fluoride; DMSO, dimethyl sulfoxide; $\mathrm{P}-\mathrm{V}$, pressure-volume

Key words: matrine, diabetic cardiomyopathy, transforming growth factor- $\beta 1$, Smads, cardiac fibroblasts, fibrosis revealed to be markedly activated; however, the expression of inhibitory Smad (I-Smad, also termed Smad7) was reduced in DbCM. Matrine administration led to a marked recovery in LV function and heart compliance by exerting inhibitory effects on TGF- $\beta 1 / \mathrm{R}-\mathrm{Smad}$ signaling pathway-induced fibrosis without affecting I-Smad. Incubation with a high concentration of glucose triggered the TGF- $\beta 1 / \mathrm{R}-\mathrm{Smad}(\operatorname{Smad} 2 / 3)$ signaling pathway and suppressed I-Smad signaling transduction in cultured cardiac fibroblasts, which led to an increase in the synthesis of collagen. After cardiac fibroblasts had been treated with matrine at non-cytotoxic concentrations without affecting I-Smad, matrine blocked TGF- $\beta 1 / \mathrm{R}-\mathrm{Smad}$ signaling transduction to repress collagen production and deposition. In conclusion, the results of the present study demonstrated that TGF- $\beta 1 /$ Smad signaling-associated cardiac fibrosis is involved in the impairment of heart compliance and LV dysfunction in DbCM. By exerting therapeutic effects against cardiac fibrosis via its influence on TGF- $\beta 1 /$ Smad signaling, matrine exhibited cardioprotective effects in DbCM.

\section{Introduction}

The incidence and mortality of diabetes have been increasing rapidly in recent decades worldwide (1). The mortality of diabetes is predominantly due to its severe cardiovascular complications (2). Diabetic cardiomyopathy (DbCM) is one of the major clinical manifestations of diabetic cardiovascular complications, which is characterized by cardiac dysfunction and arrhythmia (3). As one of the most noteworthy pathological features of DbCM, cardiac fibrosis leads to cardiac remodeling, cardiac dilatation and congestive heart failure (4).

The essential pathological characteristic of cardiac fibrosis is an excessively produced and accumulated extracellular matrix (ECM). By synthesizing ECM, cardiac fibroblasts (CFs) serve a central role in inducing and developing cardiac fibrosis (5). The transforming growth factor- $\beta 1$ (TGF- $\beta 1$ ) pathway induces organ fibrosis by mediating its downstream effectors, which are a family of proteins termed Smads. Activated Smads form complexes and mediate nuclear translocation, which leads to initiation of the transcription of 
genes encoding the ECM (6). It has also been reported that the proliferation of the ECM, and ECM synthesis, were increased in human CFs incubated under high-glucose conditions (7). However, the exact molecular mechanism has yet to be fully elucidated. Inhibition of CFs may be an effective strategy for the treatment of cardiac fibrosis of DbCM.

Matrine, the active molecule of Sophora alopecuroides L. (formula: $\mathrm{C}_{15} \mathrm{H}_{24} \mathrm{~N}_{2} \mathrm{O}$ ), has been gathering interest in the research community due to its potent and various biological activities. Results from a previous study published by the present authors indicated that matrine improved the left ventricular (LV) function of animals in an experimental DbCM model (8). The anti-fibrotic activity of matrine on organ fibrosis via inhibition of the TGF- $\beta /$ Smad pathway has also been suggested by another previously published study (9). In the present study, the anti-fibrotic effect of matrine on cardiac fibrosis was investigated with an experimental DbCM model. In the in vivo part of the study, the effects of matrine on cardiac function and compliance were studied. In the in vitro part, the possible molecular mechanisms involved in the anti-fibrotic activity of matrine were investigated. The results of the present study should add further to our in-depth understanding of the pathological mechanism of cardiac fibrosis associated with DbCM. Furthermore, the results from this study should provide a theoretical foundation for the application of matrine-based compounds in DbCM treatment.

\section{Materials and methods}

Animal experimental protocol. An experimental animal model of DbCM was induced as described previously in 7-week oldmale and female (1:1) Sprague-Dawley rats (weight, 180-220 g) provided by Animal Experimental Center of Xi'an Jiaotong University (10). The rats were raised in independent cages in a 12-h light/dark cycle, $25 \pm 2{ }^{\circ} \mathrm{C}$ and $50 \%$ humidity. Animals had access to standard chow and clean water ad libitum. Intraperitoneal injection of streptozotocin (STZ; $65 \mathrm{mg} / \mathrm{kg}$ body weight) was employed to induce diabetes in rats. Prior to the induction of diabetes, rats were administered with matrine (Sigma-Aldrich; Merck KGaA, Darmstadt, Germany) orally at a dose of $300 \mathrm{mg} /$ body weight per day for 10 days. Further details of the treatments, and the treatment groups, are provided in Table I. In the present study, all animal experimental procedures were approved by the Experimental Animal Use Ethics Committee of Xi'an Jiaotong University, and were performed in accordance with guidelines for the Care and Use of Laboratory Animals issued by the Chinese Council on Animal Research.

Invasive hemodynamic evaluation of cardiac function. An invasive hemodynamic method described in our previous study (11) was employed to evaluate the cardiac functions. Briefly, following anesthetization with chloral hydrate ( $10 \% \mathrm{v} / \mathrm{v}, 0.85 \mathrm{mg} / \mathrm{kg}$ body weight), a Mikro-Tip catheter transducer (Millar Instruments, Houston, TX, USA) was intubated into the left ventricle via the right carotid artery. Signals captured by the transducer were inputted into the PowerLab 4/25 Biological Information Analysis system (ADInstruments, Dunedin, New Zealand), from which the parameters, including LV end-diastolic pressure (LVEDP),
LV systolic pressure (LVSP), positive maximal rate of LV increased pressure $\left(+\mathrm{LVdP} / \mathrm{dt}_{\max }\right)$ and negative maximal rate of $\mathrm{LV}$ increased pressure (-LVdP/dt $\mathrm{max}_{\text {max }}$, were outputted.

Cardiaccomplianceassessments.By plotting a pressure-volume (P-V) curve from the measurements, cardiac compliance was evaluated in isolated perfused hearts. $\mathrm{K}-\mathrm{H}$ buffering solution $\left(95 \% \mathrm{O}_{2} / 5 \% \mathrm{CO}_{2}\right.$ at $\left.37^{\circ} \mathrm{C}\right)$ was used to perfuse the isolated Langendorff hearts. Through left atrial appendage and mitral valves, a non-compliant balloon filled with $\mathrm{K}-\mathrm{H}$ solution was inserted into the left ventricle. The non-compliant balloon was connected to a pressure transducer (Millar Instruments), and the measurements were recorded using the PowerLab 4/25 Biological Information Analysis system (ADInstruments). LVDP was recorded when the volume of the balloon increased from 0.06 to $0.42 \mathrm{ml}$. Based on these measurements, the P-V curve was plotted.

Cardiac collagen staining. Sirius red staining was used to visualize collagens in cardiac tissue. Following harvesting and trimming, cardiac tissues were fixed in neutral buffered formalin $(10 \%, \mathrm{v} / \mathrm{v})$ at room temperature for $10 \mathrm{~h}$. Subsequently, the cardiac tissue was embedded in paraffin and sectioned into $4 \mu \mathrm{m}$-thick slices. A Picro Sirius Red stain kit (cat. no. ab150681; Abcam, Cambridge, UK) was used to treat the sections, according to the protocol provided by the manufacturer. Images were captured using a phase-contrast microscope. The addition of Sirius Red stain specifically enabled the positive staining of collagens.

Isolation of primary CFs. Primary CFs were isolated from the left ventricles of neonatal SD rats following the protocol described in a previous study (12). After having been harvested from 2-day-old SD rats, the hearts were minced and digested using type II collagenase $(120 \mathrm{U} / \mathrm{ml}$; Beijing Solarbio Science and Technology Co., Ltd., Beijing, China). The dissociated cells were collected by centrifugation $(200 \mathrm{x} g$ for $8 \mathrm{~min}$ at room temperature) and further cultured in MEM medium (Hyclone $^{\mathrm{TM}}$ ) supplemented with fetal bovine serum (FBS; $10 \%$, Hyclone $^{\mathrm{TM}}$ ) (both from GE Healthcare Life Sciences, Shanghai, China) and penicillin-streptomycin antibiotic mix (Sigma-Aldrich; Merck KGaA). Cells were incubated in a humidified incubator under conditions of $95 \%$ fresh air and $5 \% \mathrm{CO}_{2}$ at $37^{\circ} \mathrm{C}$. The adherent cells were cultured to reach over $80 \%$ confluence. In excess of $95 \%$ of the collected cells were vimentin-positive $/ \alpha$-actin-negative. Cells from passages 2-3 were used. Table II shows the details of grouping, and treatments of the isolated cells.

MTT assay. MTT assay was used to assess the cell viability of isolated CFs. After seeding into a 96-well plate, cultured $\mathrm{CFs}$ were subsequently incubated at $37^{\circ} \mathrm{C}$ with matrine at serial concentrations of $0.25,0.5,1.0,1.5,2.0$ and $2.5 \mathrm{mmol} / 1$ for $48 \mathrm{~h}$. MTT ( $5 \mathrm{mg} / \mathrm{ml})$ was then added into each well, and the cells were incubated for a further $4 \mathrm{~h}$. Dimethyl sulfoxide (DMSO) was added to dissolve the resultant formazan crystals. Measurements of absorbance at $540 \mathrm{~nm}\left(\mathrm{~A}_{540}\right)$ were recorded using a plate reader (Bio-Rad Laboratories, Inc., Hercules, CA, USA). Cell viability was defined according to the formula: $\left(\mathrm{A}_{540}\right.$ of matrine - treated cells/ $\mathrm{A}_{540}$ of control cells) $\mathrm{x} 100 \%$. 
Table I. Treatment of the rats in the different groups.

\begin{tabular}{|c|c|c|c|c|}
\hline \multirow[b]{2}{*}{ Group } & \multicolumn{2}{|c|}{ Treatment 1} & \multicolumn{2}{|c|}{ Treatment 2} \\
\hline & Reagent & Description & Reagent & Description \\
\hline Ctrl & Physiological saline & $\begin{array}{l}10 \text { days prior to injection; } \\
\text { oral administration; } \\
\text { equal volume to matrine }\end{array}$ & Sodium citrate buffer (pH 4.5) & $\begin{array}{l}\text { Equal volume to STZ } \\
\text { injection; single } \\
\text { intraperitoneal injection }\end{array}$ \\
\hline $\mathrm{DbCM}$ & Physiological saline & $\begin{array}{l}10 \text { days before injection; } \\
\text { oral administration; } \\
\text { equal volume to matrine }\end{array}$ & $\begin{array}{l}\text { STZ (dissolved in sodium } \\
\text { citrate buffer, } \mathrm{pH} 4.5 \text { ) }\end{array}$ & $\begin{array}{l}65 \mathrm{mg} / \mathrm{kg} \text { body weight; } \\
\text { single intraperitoneal } \\
\text { injection }\end{array}$ \\
\hline Mat & Matrine & $\begin{array}{l}10 \text { days before injection; } \\
\text { oral administration; } \\
200 \mathrm{mg} / \mathrm{kg} \text { /day for } \\
10 \text { consecutive days }\end{array}$ & Sodium citrate buffer, $\mathrm{pH} 4.5$ & $\begin{array}{l}\text { Equal volume to STZ } \\
\text { injection; single } \\
\text { intraperitoneal injection }\end{array}$ \\
\hline DbCM+Mat & Matrine & $\begin{array}{l}10 \text { days prior to injection; } \\
\text { oral administration; } \\
200 \mathrm{mg} / \mathrm{kg} / \text { day for } \\
10 \text { consecutive days }\end{array}$ & $\begin{array}{l}\text { STZ (dissolved in sodium } \\
\text { citrate buffer with } \mathrm{pH} 4.5 \text { ) }\end{array}$ & $\begin{array}{l}65 \mathrm{mg} / \mathrm{kg} \text { body weight; } \\
\text { single intraperitoneal } \\
\text { injection }\end{array}$ \\
\hline
\end{tabular}

Ctrl, control; Mat, matrine; STZ, streptozotocin; DbCM, diabetic cardiomyopathy.

Table II. Treatment of CFs in different groups.

Treatment 1

\begin{tabular}{ll}
\cline { 2 - 2 } Group & \multicolumn{1}{c}{ Reagent } \\
\hline Ctrl & Vehicle solution \\
HG & Vehicle solution \\
Mat & Matrine solution \\
& $(0.25,0.5,1.0,1.5$, \\
& 2.0 and $2.5 \mathrm{mmol} / \mathrm{l})$ \\
HG+Mat & Matrine solution \\
& $(0.25,0.5,1.0,1.5$, \\
& 2.0 and $2.5 \mathrm{mmol} / \mathrm{l})$
\end{tabular}

Treatment 2

\begin{tabular}{ll}
\hline \multicolumn{1}{c}{ Reagent } & \multicolumn{1}{c}{ Description } \\
\hline Normal glucose medium & Glucose concentration at $5.5 \mathrm{mmol} / 1$ \\
HG medium & Glucose concentration at $25 \mathrm{mmol} / 1$ \\
Normal glucose medium & Glucose concentration at $5.5 \mathrm{mmol} / 1$
\end{tabular}

Incubation for $48 \mathrm{~h}$ Incubation for $48 \mathrm{~h}$ Incubation for $48 \mathrm{~h}$

Incubation for $48 \mathrm{~h}$

HG medium

Glucose concentration at $25 \mathrm{mmol} / \mathrm{l}$

CFs, cardiac fibroblasts; Ctrl, control; HG, high glucose; Mat, matrine.

Western blotting. The RIPA lysis buffer system (Santa Cruz Biotechnology, Inc., Dallas, TX, USA) with phenylmethylsulfonylfluoride (PMSF; Santa Cruz Biotechnology, Inc.) was used to obtain an homogenate of harvested cardiac tissue and cultured CFs, as per the manufacturer's instructions. Following centrifugation $(12,500 \mathrm{x} \mathrm{g})$ at $4^{\circ} \mathrm{C}$ for $10 \mathrm{~min}$, the supernatants were collected. The total protein concentration was detected using a bicinchoninic acid (BCA) protein assay kit (Santa Cruz Biotechnology, Inc.). Proteins were subjected to $8 \%$ SDS-PAGE, and subsequently separated by vertical electrophoresis. The samples were then transferred to polyvinylidene fluoride (PVDF) membranes (EMD Millipore, Billerica, MA, USA) electrically at $10-12 \mathrm{~V}$ for $50 \mathrm{~min}$. Specific primary antibodies against TGF- $\beta 1$ (cat. no. SC7892, 1:1,000; Santa Cruz Biotechnology, Inc.), Smad2 (cat. no. ab40855, 1:2,000), Smad3 (cat. no. ab28379, 1:2,000) (both from Abcam), Smad7 (cat. no.PA1-41506, 1:2,000; Thermo Fisher Scientific, Inc., Waltham,
MA, USA), phospho-Smad2 (cat. no. ab53100, 1:2,000), phospho-Smad3 (cat. no. ab52903, 1:2,000) (both from Abcam), collagen I (cat. no. 5D8-G9, 1:2,000; Thermo Fisher Scientific, Inc.) and GAPDH (cat. no. ab8245, 1:5,000; Abcam) were used to incubate the membranes at $4^{\circ} \mathrm{C}$ for $12 \mathrm{~h}$. After washing, corresponding horseradish peroxidase (HRP)-conjugated secondary antibodies (cat. no. ab13168, 1:1,000; Abcam) (cat. nos. sc-2350 and sc-2371, 1:1,000; Santa Cruz Biotechnology, Inc.) were used to incubate the membranes at room temperature for $30 \mathrm{~min}$. The membranes were developed using Western Blotting Luminal reagent (Santa Cruz Biotechnology, Inc.), and the bands were visualized on X-ray film in a dark room.

Statistical analysis. Data in the present study are presented as the mean \pm standard deviation. The differences between groups were analyzed using one-way analysis of variance, and statistical analysis was performed with SPSS software, 
Table III. Hemodynamic parameters and blood glucose concentration comparisons between groups $(\mathrm{n}=3)$.

\begin{tabular}{|c|c|c|c|c|c|}
\hline Group & $\begin{array}{c}\text { Blood glucose } \\
\text { concentration }(\mathrm{mmol} / \mathrm{l})\end{array}$ & $\begin{array}{l}\text { Left ventricular } \\
\text { systolic pressure } \\
\quad(\mathrm{mmHg})\end{array}$ & $\begin{array}{l}\text { Left ventricular } \\
\text { end-diastolic } \\
\text { pressure }(\mathrm{mmHg})\end{array}$ & $\begin{array}{c}+ \text { Left ventricular } \\
{\mathrm{dP} / \mathrm{dt}_{\max }}^{(\mathrm{mmHg} / \mathrm{sec})}\end{array}$ & $\begin{array}{c}\text {-Left ventricular } \\
{\mathrm{dP} / \mathrm{dt}_{\max }}^{(\mathrm{mmHg} / \mathrm{sec})}\end{array}$ \\
\hline Ctrl & $9.25 \pm 3.32$ & $107.82 \pm 12.61$ & $22.18 \pm 5.59$ & $1,988.35 \pm 221.54$ & $2,677.16 \pm 345.27$ \\
\hline Mat & $10.15 \pm 3.10$ & $112.37 \pm 13.05$ & $23.29 \pm 5.75$ & $1,973.00 \pm 224.45$ & $2,713.05 \pm 332.19$ \\
\hline $\mathrm{DbCM}$ & $18.27 \pm 5.56^{\mathrm{a}, \mathrm{b}}$ & $68.54 \pm 9.75^{\mathrm{a}, \mathrm{b}}$ & $64.78 \pm 6.14^{\mathrm{a}, \mathrm{b}}$ & $549.43 \pm 95.18^{\mathrm{a}, \mathrm{b}}$ & $1,389.75 \pm 154.11^{\mathrm{a}, \mathrm{b}}$ \\
\hline DbCM+Mat & $19.34 \pm 4.91^{\mathrm{a}, \mathrm{b}}$ & $82.10 \pm 7.45^{\mathrm{a}-\mathrm{c}}$ & $48.20 \pm 5.61^{\mathrm{a}-\mathrm{c}}$ & $1,244.75 \pm 111.43^{\mathrm{a}-\mathrm{c}}$ & $1,799.48 \pm 177.42^{\mathrm{a}-\mathrm{c}}$ \\
\hline
\end{tabular}

${ }^{\mathrm{a}} \mathrm{P}<0.05$ vs. Ctrl; ${ }^{\mathrm{b}} \mathrm{P}<0.05$ vs. Mat; ${ }^{\mathrm{C}} \mathrm{P}<0.05$ vs. DbCM. Ctrl, control; Mat, matrine; DbCM, diabetic cardiomyopathy.

version 17.0 (SPSS, Inc., Chicago, IL, USA). P $<0.05$ was considered to indicate a statistically significant difference.

\section{Results}

Matrine attenuates left ventricular functions impairment in experimental rat model of $\mathrm{DbCM}$. The experimental rat model of $\mathrm{DbCM}$ is characterized by elevated blood glucose levels and deteriorated LV function. Matrine treatment did not change the blood glucose levels in diabetic animals. The LV functions of control animals were not affected by matrine. However, matrine administration increased LVSP, $+\mathrm{LVdP} / \mathrm{dt}_{\max }$ and $-\mathrm{LVdP} / \mathrm{dt}_{\max }$, whereas LVEDP was decreased in the experimental rat model of DbCM. These results were demonstrated in Table III.

Matrine recovers cardiac compliance loss in experimental rat model of DbCM. As demonstrated in the P-V curve in Fig. 1, the LV compliance was markedly impaired in isolated hearts from rats with $\mathrm{DbCM}$. These changes were demonstrated by the significant P-V curve leftward shift. However, the P-V curve of isolated hearts from the $\mathrm{DbCM}+$ Mat group displayed a significant rightward shift, indicating markedly recovered LV compliance.

Matrine inhibits cardiac fibrosis induced by the activation of the TGF- $\beta 1 /$ Smad signaling pathway in rats with $D b C M$. Sirius red staining was used to evaluate the extent of cardiac fibrosis, and captured images are shown in Fig. 2A. Compared with the Ctrl and Mat groups, the optical density of Sirius Red staining increased markedly in the DbCM group, suggesting the occurrence of aggravated cardiac fibrosis. It was also observed that the optical density of Sirius Red staining decreased markedly in the $\mathrm{DbCM}+$ Mat group, indicating that matrine treatment alleviated cardiac fibrosis. Fig. 2B demonstrates the effects of matrine on the TGF- $\beta 1 /$ Smad signaling pathway. Compared with Ctrl and Mat, the protein expression level of TGF- $\beta 1$ increased in the $\mathrm{DbCM}$ group, but was markedly reduced by matrine administration in the DbCM group. Correspondingly, compared with Ctrl and Mat, the phosphorylation levels of $\mathrm{Smad} 2$ and 3 were also revealed to be elevated in $\mathrm{DbCM}$, indicating activation of the TGF- $\beta 1 /$ Smad signaling pathway. By contrast, matrine treatment markedly decreased the expression level of TGF- $\beta 1$, and further inhibited phosphorylation of Smad2 and Smad3 in the DbCM+Mat group. As a result, the expression level of collagen I increased markedly in the

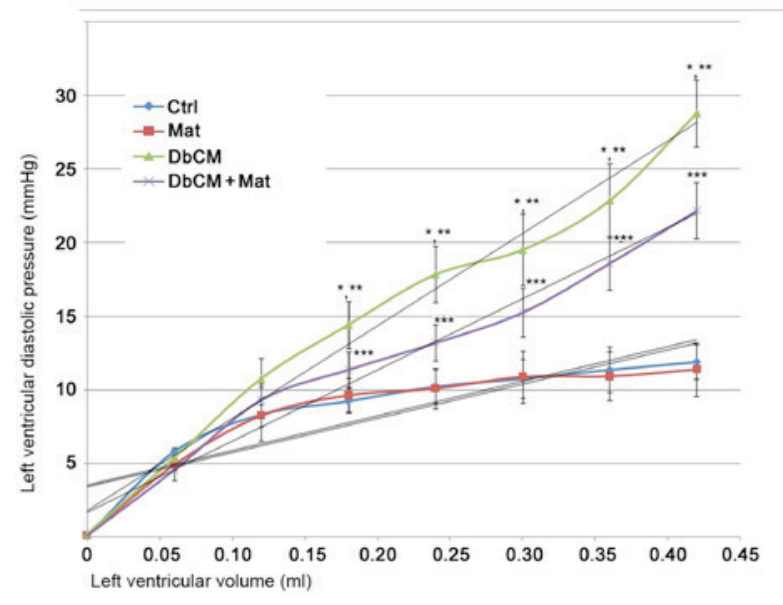

Figure 1. Cardiac compliance detection and plotting in isolated hearts The line chart in this figure demonstrates the P-V (left ventricular diastolic pressure-left ventricular volume) curve of isolated hearts from the Ctrl, Mat, $\mathrm{DbCM}$ and $\mathrm{DbCM}+\mathrm{Mat}$ groups. Results are presented as the mean \pm standard deviation. Approximately linear trends are shown. ${ }^{*} \mathrm{P}<0.05$ vs. $\mathrm{Ctrl} ;{ }^{* *} \mathrm{P}<0.05$ vs. Mat; ${ }^{* * *} \mathrm{P}<0.05$ vs. DbCM. Ctrl, control; Mat, matrine; DbCM, diabetic cardiomyopathy.

$\mathrm{DbCM}$ group, but decreased in the $\mathrm{DbCM}+\mathrm{Mat}$ group. The expression level of inhibitory Smad (I-Smad), namely Smad7, was shown to be decreased in the $\mathrm{DbCM}$ group, although this was not significantly affected by matrine treatment.

Matrine at low concentrations exerts no inhibitory effects on cell proliferation of CFs incubated under high-glucose conditions. As shown in Fig. 3, the cell viability was assessed by MTT assay. The high-glucose-incubated CFs were treated with matrine at serial concentrations. Starting at $2.0 \mathrm{mmol} / \mathrm{l}$, the viability of the CFs was significantly decreased. Therefore, matrine at concentrations of $0.25,0.5$ and $1.0 \mathrm{mmol} / \mathrm{l}$ were identified as the non-cytotoxic concentrations that were selected for subsequent experiments.

Matrine treatment inhibits collagen synthesis by suppressing activation of the TGF- $\beta 1 /$ Smad signaling pathway in high-glucose-incubated CFs. As shown in Fig. 4, the effects of matrine on the TGF- $\beta 1 /$ Smad signaling pathway in cultured CFs were also investigated in the present study. The expression of TGF- $\beta 1$, as well as Smad 2 and 3 phosphorylation levels, were significantly increased in the CFs incubated under high glucose conditions. Matrine treatment decreased 
A

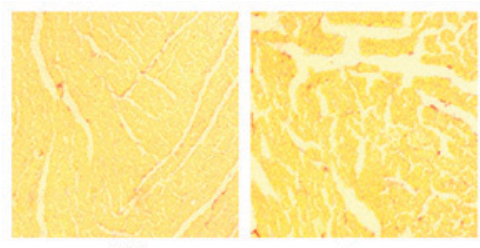

Ctrl

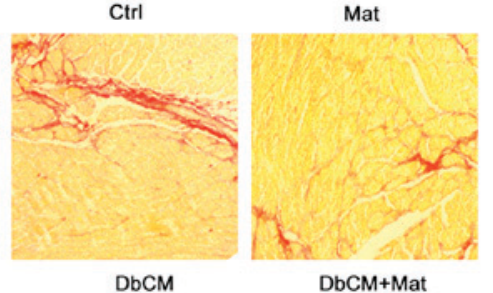

B

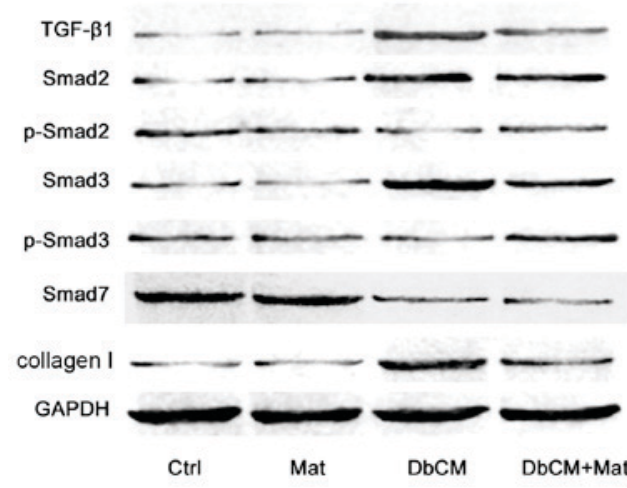

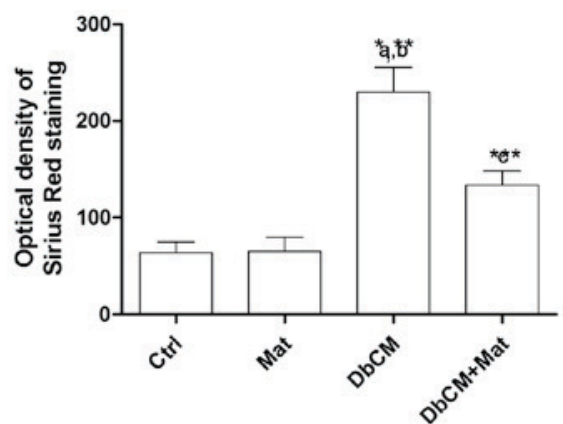

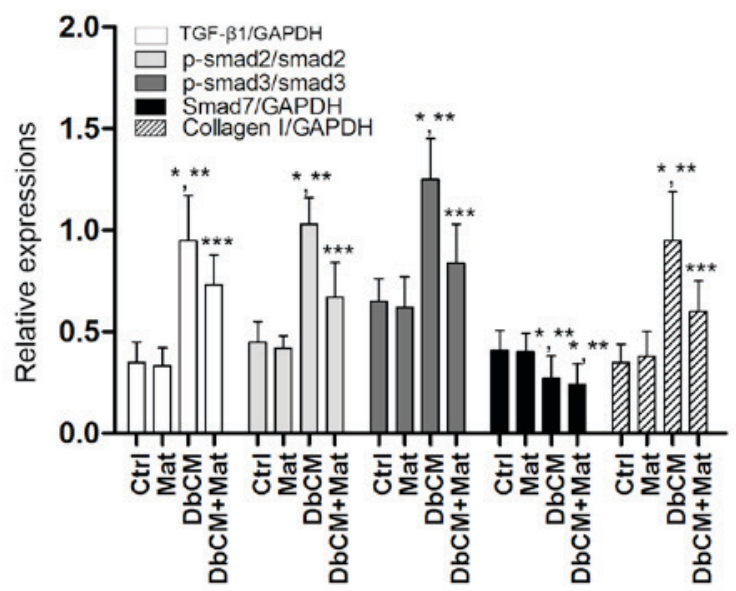

Figure 2. Collagen deposition and TGF- $\beta 1 /$ Smad signaling pathway activation in cardiac tissue. (A) In the left part of the figure, captured images of Sirius Red staining of cardiac tissue harvested from each group (Ctrl, Mat, DbCM and DbCM+Mat) are shown. The bar chart on the right shows the values of the optical densities of Sirius Red in each group (results are presented as the mean \pm standard deviation). (B) Immunoblots of TGF- $\beta 1$, Smad2, phospho (p)-Smad2, Smad3, p-Smad3, Smad7, collagen I and the loading control, GAPDH, are shown. The bar chart on the right shows the relative expression levels of TGF- $\beta 1$ (TGF- $31 /$ GAPDH), Smad7 (Smad7/GAPDH), phosphorylated Smad2 (phospho-Smad2/Smad2), phosphorylated Smad3 (phospho-Smad3/Smad3) and collagen I (collagen I/GAPDH). ${ }^{*} \mathrm{P}<0.05$ vs. Ctrl; ${ }^{* *} \mathrm{P}<0.05$ vs. Mat; ${ }^{* * *} \mathrm{P}<0.05$ vs. DbCM. TGF- $\beta 1$, transforming growth factor- $\beta 1$. Ctrl, control; Mat, matrine; DbCM, diabetic cardiomyopathy.

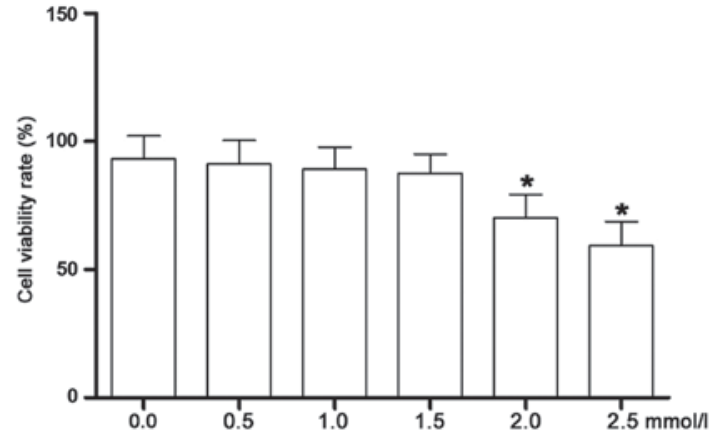

Figure 3. Cell viability of isolated CFs incubated with matrine at different concentrations. This figure demonstrates the results of the MTT assay, which determined the cell viability rate $(\%)$ of isolated CFs incubated with matrine at concentrations of $0.0,0.5,1.0,1.5,2.0$ and $2.5 \mathrm{mmol} / 1$. ${ }^{*} \mathrm{P}<0.05$ vs. the previous concentration in the series. $\mathrm{CF}$, cardiac fibroblast.

the levels of TGF- $\beta 1$ and phosphorylated $\operatorname{Smad} 2 / 3$ in a concentration-dependent manner. As a result, also in a concentration-dependent manner, matrine decreased collagen I expression in high-glucose-incubated CFs. Exposure to a high concentration of glucose suppressed the expression of Smad7, although this was not affected by matrine.

\section{Discussion}

Diabetic macro- and micro-vascular complications are the predominant causes of mortality due to diabetes. DbCM is one of the principal clinical manifestations of diabetic cardiovascular complications. DbCM is classified as a heart disease independent from other heart diseases, including congenital heart disease, coronary artery heart disease and valvular heart disease (13). DbCM is characterized by cardiac remodeling, congestive heart failure and arrhythmias (14).

Cardiac fibrosis is one of the essential pathological characteristics of cardiac remodeling, leading to cardiac structural and functional alterations (15). It was considered that cardiac fibrosis was associated with cardiac dysfunction and cellular metabolic abnormalities attributable to diabetes $(16,17)$. Clearly evident cardiac fibrosis has been identified in diabetic hearts, according to previous studies (18). CFs are activated in diabetic hearts, and exert a vital role in regulating cardiac extracellular matrix synthesis and deposition (5), causing cardiac dysfunction and myocardium stiffening (19). In the present study, it was revealed that the LV functions were significantly impaired in rats with DbCM. The chamber stiffness markedly increased in hearts isolated from rats with DbCM. 
A

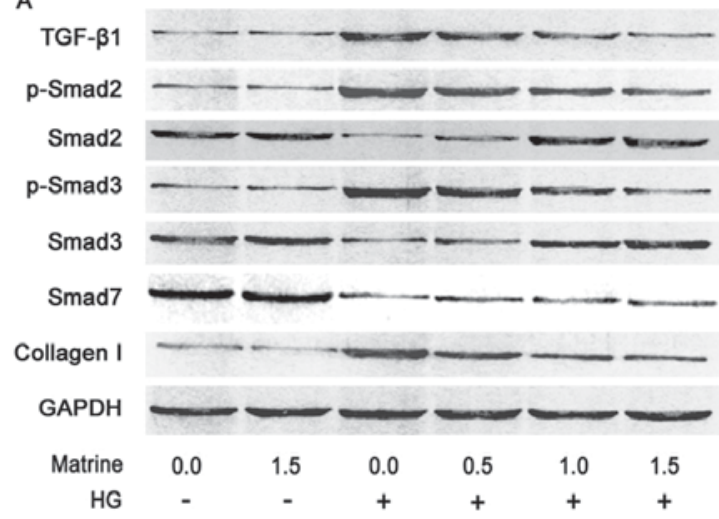

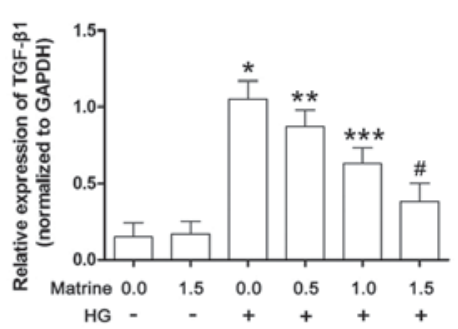

D

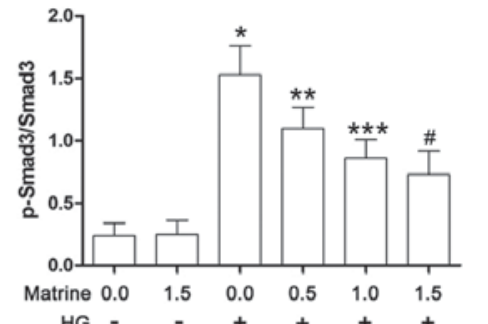

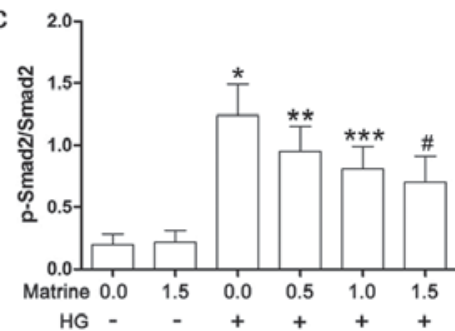

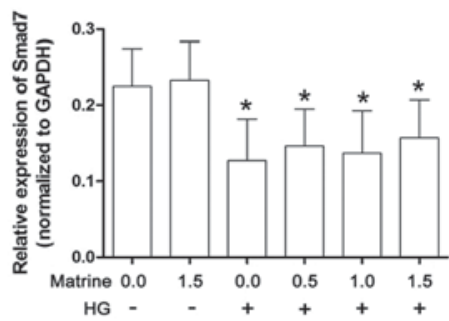

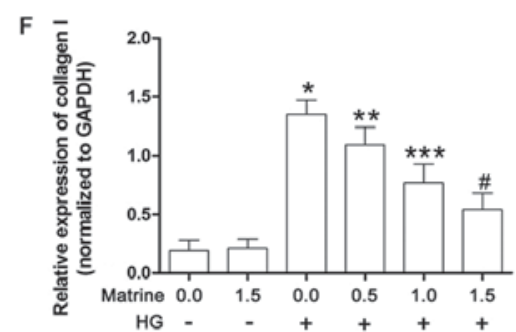

Figure 4. TGF- $\beta 1 /$ Smad signaling pathway activation in isolated cardiac fibroblasts. (A) Immunoblots of TGF- $\beta 1$, Smad2, phospho (p)-Smad2, Smad3, p-Smad3, Smad7 and GAPDH in isolated cardiac fibroblasts treated with serial concentrations of matrine $(0.0,0.5,1.0,1.5,2.0$ and $2.5 \mathrm{mmol} / \mathrm{l})$ and/or $\mathrm{HG}$ medium are shown. (B) The bar chart shows the relative expression levels of TGF- $\beta 1$ (TGF- $\beta 1 /$ GAPDH). (C) Bar charts also show the phosphorylation of Smad2 (p-Smad2/Smad2), (D) the phosphorylation of Smad3 (p-Smad3/Smad3), (E) the relative expression level of Smad7 (Smad7/GAPDH), and (F) the relative expression level of collagen I (collagen I/GAPDH). Data shown in B-F represent the mean \pm standard deviation. ${ }^{*} \mathrm{P}<0.05$ vs. first column; ${ }^{* * *} \mathrm{P}<0.05$ vs. third column; ${ }^{* * *} \mathrm{P}<0.05$ vs. fourth column; ${ }^{\#} \mathrm{P}<0.05$ vs. fifth column. TGF- $\beta 1$, transforming growth factor- $\beta 1$.

Many diabetic pathological products, including catecholamines, endothelin, insulin- like growth factor-1, advanced glycation end products and TGF- $\beta 1$, have been correlated with organ fibrosis (20-22). The molecular mechanism of cardiac fibrosis in $\mathrm{DbCM}$ has yet to be fully elucidated. Previous studies indicated that the TGF- $\beta 1$-induced signaling pathway exerts an important participatory role in cardiac fibrosis under certain cardiac pathological conditions $(23,24)$. As transcription factors, Smads are the downstream effectors of TGF- $\beta 1$, which initiate transcription of genes encoding ECM components $(25,26)$. Studies have demonstrated that activation of the TGF- $\beta 1 /$ Smad signaling pathway is responsible for hepatic, pulmonary and pancreatic fibrosis (6).

Specifically, the R-Smads (generally Smad2 and 3) are activated following phosphorylation (27). The I-Smad, also termed Smad7, suppresses the phosphorylation of Smad2/3 to inhibit the initiation of downstream target-gene transcription $(6,25)$. In the present study, in hearts harvested from rats with DbCM, the expression level of TGF- $\beta 1$ and phosphorylation levels of Smad2/3 were increased markedly, indicating that the TGF- $\beta 1 /$ Smad signaling pathway was activated. At the same time, expression of Smad7 was shown to be decreased in diabetic hearts, which also contributed to the activation of TGF- $\beta 1 / \mathrm{Smad}$ signaling. In CFs incubated under high glucose conditions, the TGF- $\beta 1 /$ Smad signaling pathway was also shown to be activated, as revealed by increased TGF- $\beta 1$ expression, decreased Smad7 expression and elevated levels of $\operatorname{Smad} 2 / 3$ phosphorylation. As a result, the synthesis of collagen I was shown to beincreased in vivo and in vitro.

Extracted from the Chinese medical herb Kushen (Sophora alopecuroides L.), matrine has been demonstrated to be one of the most important bioactive pharmacological components (28-30). Matrine was shown to affect the bioactivities of immune regulation, anti-inflammation and antioxidation $(8,31)$. Prompted by a study on the anti-fibrotic effect of matrine in several organs, including liver and kidneys (29), the present authors chose to investigate the effects of matrine on cardiac fibrosis in the present study. It was shown that ECM synthesis and deposition were suppressed in a rat model of $\mathrm{DbCM}$ where cardiac fibrosis was clearly in evidence. Matrine administration attenuated cardiac chamber stiffness, and thus the LV systolic and diastolic functions were improved.

According to previous studies, TGF- $\beta 1 /$ Smad signaling activation in CFs was shown to induce cardiac fibrosis. Several agents, including efonidipine and tanshinone, inhibited cardiac fibrosis by inhibiting this pathway $(32,33)$. In the present study, the potential molecular mechanism of matrine's anti-fibrosis effect in DbCM was investigated. The in vivo data revealed that matrine administration inhibited activation of the TGF- $\beta 1 /$ Smad signaling pathway by suppressing the expression of TGF- $\beta 1$ and phosphorylation of Smad2/3 in diabetic hearts. As a result, the accumulation of collagen I in myocytes was attenuated. However, the expression of the I-Smad, Smad7, was not shown to be affected by 
matrine. In the in vitro part of the present study, matrine at low concentrations (i.e., in order to avoid its cytotoxicity) was used to treat the high-glucose-incubated CFs. The results suggested that matrine at non-toxic concentrations deactivated the TGF- $\beta 1 / \mathrm{Smad}$ signaling pathway by suppressing the expression of TGF- $\beta 1$ and phosphorylation of $\mathrm{Smad} 2 / 3$ in a concentration-dependent manner, rather than affecting the expression of Smad7. Hence, the synthesis of collagen I in CFs was correspondingly reduced.

In conclusion, the present study has shown that a loss of cardiac compliance and function are the features of DbCM characterized by cardiac fibrosis. Secondly, the TGF- $\beta 1 / \mathrm{Smad}$ signaling pathway was activated to induce fibrosis in diabetic hearts and CFs incubated under high glucose conditions. Thirdly, matrine was shown to exert anti-fibrosis effects to improve cardiac compliance and function by suppressing activation of the TGF- $\beta 1 /$ Smad signaling pathway in CFs in DbCM.

\section{Acknowledgements}

This study was supported by the National Scientific Foundation of China (grant no. 81500308), the China Postdoctoral Science Foundation (grant no. 2016M590956) and the Sailing Foundation (grant no. LHJJ20159029).

\section{References}

1. Echouffo-Tcheugui JB and Dagogo-Jack S: Preventing diabetes mellitus in developing countries. Nat Rev Endocrinol 8: 557-562, 2012.

2. Lotfy M, Adeghate J, Kalasz H, Singh J and Adeghate E: Chronic complications of diabetes mellitus: A mini review. Curr Diabetes Rev 13: 3-10, 2017.

3. Boudina $S$ and Abel ED: Diabetic cardiomyopathy revisited. Circulation 115: 3213-3223, 2007.

4. Westermeier F, Riquelme JA, Pavez M, Garrido V, Díaz A, Verdejo HE, Castro PF, García L and Lavandero S: New molecular insights of insulin in diabetic cardiomyopathy. Front Physiol 7: 125, 2016.

5. Travers JG, Kamal FA, Robbins J, Yutzey KE and Blaxall BC: Cardiac fibrosis: The fibroblast awakens. Circ Res 118: 1021-1040, 2016.

6. Xu F, Liu C, Zhou D and Zhang L: TGF-beta/SMAD pathway and its regulation in hepatic fibrosis. J Histochem Cytochem 64 157-167, 2016.

7. Song SE, Kim YW, Kim JY, Lee DH, Kim JR and Park SY: IGFBP5 mediates high glucose-induced cardiac fibroblast activation. J Mol Endocrinol 50: 291-303, 2013.

8. Liu ZW, Wang JK, Qiu C, Guan GC, Liu XH, Li SJ and Deng ZR: Matrine pretreatment improves cardiac function in rats with diabetic cardiomyopathy via suppressing ROS/TLR-4 signaling pathway. Acta Pharmacol Sin 36: 323-333, 2015.

9. Gao HY, Li GY, Lou MM, Li XY, Wei XY and Wang JH: Hepatoprotective effect of Matrine salvianolic acid B salt on carbon tetrachloride-induced hepatic fibrosis. J Inflamm (Lond) 9: 16, 2012.

10. Liu Z, Zhao N, Zhu H, Zhu S, Pan S, Xu J, Zhang X, Zhang Y and Wang J: Circulating interleukin-1 $\beta$ promotes endoplasmic reticulum stress-induced myocytes apoptosis in diabetic cardiomyopathy via interleukin-1 receptor-associated kinase- 2 . Cardiovasc Diabetol 14: 125, 2015.

11. Liu Z, Cai H, Zhu H, Toque H, Zhao N, Qiu C, Guan G, Dang Y and Wang J: Protein kinase RNA-like endoplasmic reticulum kinase (PERK)/calcineurin signaling is a novel pathway regulating intracellular calcium accumulation which might be involved in ventricular arrhythmias in diabetic cardiomyopathy. Cell Signal 26: 2591-2600, 2014.

12. Li R, Xiao J, Qing X, Xing J, Xia Y, Qi J, Liu X, Zhang S, Sheng X, Zhang X and Ji X: Sp1 mediates a therapeutic role of $\mathrm{miR}-7 \mathrm{a} / \mathrm{b}$ in angiotensin II-induced cardiac fibrosis via mechanism involving the Tgf- $\beta$ and mapks pathways in cardiac fibroblasts. PLoS One 10: e0125513, 2015.
13. Adeghate E: Molecular and cellular basis of the aetiology and management of diabetic cardiomyopathy: A short review. Mol Cell Biochem 261: 187-191, 2004.

14. Stratmann B, Worms J and Tschoepe D: Diabetic cardiomyopathy/heart failure: News regarding etiology, diagnosis, therapy. Dtsch Med Wochenschr 139: 2006-2009, 2014 (In German).

15. Fan D, Takawale A, Lee J and Kassiri Z: Cardiac fibroblasts, fibrosis and extracellular matrix remodeling in heart disease. Fibrogenesis Tissue Repair 5: 15, 2012.

16. Xie Y, Liao J, Li M, Wang X, Yang Y, Ge J, Chen R and Chen H: Impaired cardiac microvascular endothelial cells function induced by Coxsackievirus B3 infection and its potential role in cardiac fibrosis. Virus Res 169: 188-194, 2012.

17. Cavalera M, Wang J and Frangogiannis NG: Obesity, metabolic dysfunction, and cardiac fibrosis: Pathophysiological pathways, molecular mechanisms and therapeutic opportunities. Transl Res 164: 323-335, 2014.

18. Russo I and Frangogiannis NG: Diabetes-associated cardiac fibrosis: Cellular effectors, molecular mechanisms and therapeutic opportunities. J Mol Cell Cardiol 90: 84-93, 2016.

19. Hutchinson KR, Lord CK, West TA and Stewart JA Jr: Cardiac fibroblast-dependent extracellular matrix accumulation is associated with diastolic stiffness in type 2 diabetes. PLoS One 8: e72080, 2013

20. Chen M, Li H, Wang G, Shen X, Zhao S and Su W: Atorvastatin prevents advanced glycation end products (AGEs)-induced cardiac fibrosis via activating peroxisome proliferator-activated receptor gamma (PPAR- $\gamma$ ). Metabolism 65: 441-453, 2016.

21. Bos R, Mougenot N, Findji L, Médiani O, Vanhoutte PM and Lechat P: Inhibition of catecholamine-induced cardiac fibrosis by an aldosterone antagonist. J Cardiovasc Pharmacol 45: 8-13, 2005.

22. Liang M, Woodard LE, Liang A, Luo J, Wilson MH, Mitch WE and Cheng J: Protective role of insulin-like growth factor-1 receptor in endothelial cells against unilateral ureteral obstruction-induced renal fibrosis. Am J Pathol 185: 1234-1250, 2015.

23. Li FZ, Cai PC, Song LJ, Zhou LL, Zhang Q, Rao SS, Xia Y, Xiang F, Xin JB, Greer PA, et al: Crosstalk between calpain activation and TGF- $\beta 1$ augments collagen-I synthesis in pulmonary fibrosis. Biochim Biophys Acta 1852: 1796-1804, 2015.

24. Cutroneo KR, White SL, Phan SH and Ehrlich HP: Therapies for bleomycin induced lung fibrosis through regulation of TGF-beta1 induced collagen gene expression. J Cell Physiol 211: 585-589, 2007.

25. Meng XM, Tang PM, Li J and Lan HY: TGF- $\beta /$ Smad signaling in renal fibrosis. Front Physiol 6: 82, 2015.

26. Zeglinski MR, Hnatowich M, Jassal DS and Dixon IM: SnoN as a novel negative regulator of TGF- $\beta /$ Smad signaling: A target for tailoring organ fibrosis. Am J Physiol Heart Circ Physiol 308: H75-H82, 2015.

27. Tasanarong A, Kongkham S, Duangchana S, Thitiarchakul S and Eiam-Ong S: Vitamin E ameliorates renal fibrosis by inhibition of TGF-beta/Smad2/3 signaling pathway in UUO mice. J Med Assoc Thai 94 (Suppl 7): S1-S9, 2011.

28. Huang J and Xu H: Matrine: Bioactivities and structural modifications. Curr Top Med Chem 16: 3365-3378, 2016.

29. Yu JL, Li JH, Chengz RG, Ma YM, Wang XJ and Liu JC: Effect of matrine on transforming growth factor $\beta 1$ and hepatocyte growth factor in rat liver fibrosis model. Asian Pac J Trop Med 7: 390-393, 2014

30. Yu J, Yang S, Wang X and Gan R: Matrine improved the function of heart failure in rats via inhibiting apoptosis and blocking $\beta 3$ adrenoreceptor/endothelial nitric oxide synthase pathway. Mol Med Rep 10: 3199-3204, 2014.

31. Zhang L, Zhang H, Zhu Z, Lu X, Zhou M, Sun X, He L, Bai Y and Ma L: Matrine regulates immune functions to inhibit the proliferation of leukemic cells. Int J Clin Exp Med 8: 5591-5600, 2015.

32. Lei B, Hitomi H, Mori T, Nagai Y, Deguchi K, Mori H, Masaki T, Nakano D, Kobori H, Kitaura Y and Nishiyama A: Effect of efonidipine on TGF- $\beta 1$-induced cardiac fibrosis through Smad2-dependent pathway in rat cardiac fibroblasts. J Pharmacol Sci 117: 98-105, 2011.

33. Zhan CY, Tang JH, Zhou DX and Li ZH: Effects of tanshinone IIA on the transforming growth factor $\beta 1 /$ Smadsignaling pathway in rat cardiac fibroblasts. Indian J Pharmacol 46: 633-638, 2014.

This work is licensed under a Creative Commons Attribution-NonCommercial-NoDerivatives 4.0 International (CC BY-NC-ND 4.0) License. 